\title{
Two-dimensional repulsive Fermi polarons with short- and long-range interactions
}

\author{
Raúl Bombín $\odot,{ }^{1,2, *}$ Tommaso Comparin, ${ }^{2, \dagger}$ Gianluca Bertaina, ${ }^{3}$ Ferran Mazzanti, ${ }^{1}$ \\ Stefano Giorgini, ${ }^{2}$ and Jordi Boronat ${ }^{1}$ \\ ${ }^{1}$ Departament de Física, Universitat Politècnica de Catalunya, Campus Nord B4-B5, E-08034 Barcelona, Spain \\ ${ }^{2}$ INO-CNR BEC Center and Dipartimento di Fisica, Università di Trento, 38123 Povo, Italy \\ ${ }^{3}$ Dipartimento di Chimica and Dipartimento di Fisica, Università degli Studi di Milano, Via Golgi 19, 20133 Milano, Italy
}

(Received 24 May 2019; published 9 August 2019)

\begin{abstract}
We study the repulsive polaron problem in a two-component two-dimensional system of fermionic atoms. We use two different interaction models: a short-range (hard-disk) potential and a dipolar potential. In our approach, all the atoms have the same mass and we consider the system to be composed of a uniform bath of a single species and a single atomic impurity. We use the diffusion Monte Carlo method to evaluate polaron properties such as its chemical potential and pair distribution functions, together with a discussion on the deficit of volume induced by the impurity. We also evaluate observables that allow us to determine the validity of the quasiparticle picture: the quasiparticle residue and the effective mass of the polaron. Employing two different potentials allows us to identify the universality regime, where the properties depend only on the gas parameter $n a_{s}^{2}$ fixed by the bath density and the two-dimensional scattering length.
\end{abstract}

DOI: 10.1103/PhysRevA.100.023608

\section{INTRODUCTION}

The polaron problem was put forward by Landau and Pekar $[1,2]$ to study the interaction of an electron with a crystal lattice. In the strongly coupled regime, it was shown that the distortion of the lattice, caused by the presence of the electron, may induce a local potential that traps the electron. Some years later, Fröhlich developed a Hamiltonian formulation [3] to describe the coupling between the impurity (electron) and the phonon modes. Using this model, a first variational ground-state solution for the intermediate coupling regime was derived by Feynman [4]. Some decades later, the picture was completed with exact results for the Fröhlich model Hamiltonian obtained by using the diagrammatic quantum Monte Carlo (QMC) method [5,6]. The polaron (impurity) problem has also been studied in other fields of physics such as condensed matter (cf. an impurity of ${ }^{3} \mathrm{He}$ in bulk ${ }^{4} \mathrm{He}[7,8]$ ) and nuclear matter [9].

The achievement of the Bose-Einstein condensate state (BEC) in recent decades has provided a platform for the study of the polaron. The name Bose polaron was coined to indicate an impurity coupled to a BEC, and two-component mixtures of ultracold gases featuring a very small concentration of one of the components were proposed as candidate systems in which to investigate the quasiparticle nature of the impurities $[10,11]$. In recent years, these configurations have been realized in mixtures of both different hyperfine levels of the same atomic species [12] and of different atoms [13,14]. In these experiments, the polaron problem was investigated close to a Feshbach resonance, which allows for the tunability of the interaction strength between the impurity and the bath. Two

\footnotetext{
*raul.bombin@upc.edu

†tommaso.comparin@unitn.it
}

branches have been characterized at very low temperatures: the attractive polaron branch, corresponding to the ground state of the impurity in the medium, and the repulsive polaron branch, which consists in an excited state of the impurity, where the effective interaction between the impurity and the bath is repulsive $[15,16]$.

Furthermore, in the context of ultracold gases, Fermi degenerate systems offer new possibilities where the polaron picture can arise. Experimental measurements have been reported for a spin-down impurity "dressed" in a bath of a spin-up Fermi gas (cf. ${ }^{6} \mathrm{Li} \mathrm{[17])} \mathrm{and} \mathrm{for} \mathrm{atom} \mathrm{mixtures} \mathrm{such}$ as ${ }^{40} \mathrm{~K}$ impurities into ${ }^{6} \mathrm{Li}$, where attractive and repulsive polaron branches have also been observed [18]. While the relation between the bosonic case and the Fröhlich formulation is straightforward, the fermionic equivalent problem (Fermi polaron) is more challenging and opens the door to a richer scenario. Some theoretical works [19-21] have studied the polaron as a first insight into some physical phenomena that are characteristic of the strongly interacting regime: the pairing mechanism that gives rise to the BECBCS crossover [17,22,23], possible itinerant ferromagnetism in two-component systems [24-27], or the Kondo effect in systems containing magnetic impurities [28].

The realization of quantum degenerate systems composed of atoms with large magnetic moment has motivated additional interest in the polaron problem. The dominant dipolar interaction between these atoms is of longer range and is anisotropic. This was first achieved with $\mathrm{Cr}$ atoms [29,30] and more recently also with Dy [31,32] and Er [33,34] that have a larger magnetic moment than $\mathrm{Cr}$. Regarding the polaron problem, the report of experimentally accessible ultracold mixtures of Er and Dy [35] and the study of low concentration impurities of ${ }^{163}$ Dy in a ${ }^{164}$ Dy droplet [36] have motivated the study of the dipolar polaron in three-dimensional [37] and quasi-two-dimensional [38] configurations. The dipolar 
polaron has also been studied in a bilayer geometry, where localization effects are predicted near the crystallization point [39].

In two dimensions (2D), quantum correlations are enhanced compared to the three-dimensional (3D) case. While the one-particle-one-hole picture has demonstrated its utility to study the Fermi polaron problem in 3D systems [19], it fails when trying to accurately reproduce the physics of the equivalent system in 2D [40]. Up to now, some efforts have been put into the study of the repulsive Fermi polaron, studied as the repulsive branch of a system with short-range interactions (cf. Refs. [41,42] and [40,43,44] for experiment and theory, respectively). However, the equivalent system but with dipolar interactions, which in principle would be accessible in current experiments, remains unexplored.

In this work, we study the repulsive Fermi polaron of a two-component system, labeled as $\uparrow$ and $\downarrow$ in analogy with spin-1/2 particles. The system, consisting of $N=N_{\uparrow}+1$ particles, contains a single atomic impurity immersed in a bath composed of $N_{\uparrow}$ atoms. We study this model with two different types of interparticle interaction, which allows us to determine the universality of the system. The first model considers that the only interaction present in the system is a short-range one between the up and down particles, modeled as a hard-disk potential, while the bath is considered to be an ideal Fermi gas. The second one assumes dipolar interactions between all the particles. In the latter case, we assume that all the dipolar moments are polarized along the direction perpendicular to the plane of motion, so that the interaction between them is isotropic (see for instance Ref. [27]).

The paper is organized as follows. In Sec. II, we report the two models that we use to describe the two-dimensional system composed of a single impurity in a polarized Fermi bath. Section III discusses the diffusion Monte Carlo method that we use for the calculations. In Sec. IV, we show our QMC results for the polaron energy and the pair correlation function between the bath and the impurity, together with an analysis of the deficit of volume induced by the impurity. We also validate the quasiparticle picture by evaluating the quasiparticle residue and the effective mass of the polaron. Finally, Sec. V contains the main conclusions of our work, emphasizing the limits of universality of the 2D Fermi polaron problem.

\section{MODELS}

We describe a two-component Fermi system in 2D. The system is composed by $N=N_{\uparrow}+1$ atoms of equal mass $m$, representing a single-component bath with one additional atomic impurity. To reproduce the physics of a uniform infinite system, we put all the particles in a square box with periodic boundary conditions, with the box side $L$ fixed by the density $n$ of the bath $\left(L=\sqrt{N_{\uparrow} / n}\right)$. The $N$-particle Hamiltonian reads

$\hat{H}=-\frac{\hbar^{2}}{2 m} \nabla_{\downarrow}^{2}-\frac{\hbar^{2}}{2 m} \sum_{i=1}^{N_{\uparrow}} \nabla_{i}^{2}+\sum_{i<j}^{N_{\uparrow}} V^{\text {bath }}\left(r_{i j}\right)+\sum_{j=1}^{N_{\uparrow}} V^{\text {int }}\left(r_{\downarrow j}\right)$, where $r_{i j} \equiv\left|\mathbf{r}_{i}-\mathbf{r}_{j}\right|$ is the distance between two bath particles and $r_{\downarrow j} \equiv\left|\mathbf{r}_{\downarrow}-\mathbf{r}_{j}\right|$ is the distance between a bath particle at $\mathbf{r}_{j}$ and the impurity position $\mathbf{r}_{\downarrow}$. Throughout this work, labels $i$ and $j$ refer to bath particles. $V^{\text {bath }}(r)$ is the two-body potential between the bath particles, and $V^{\text {int }}(r)$ is the interaction potential between the impurity and the bath. In the following, we describe the two different interaction models that we study.

\section{A. Hard-disk model}

We first consider a hard-disk model for the repulsive polaron, which is experimentally relevant for the description of the upper metastable branch of the Fermi polaron [24]. In this case, the bath is noninteracting $\left[V^{\text {bath }}(r)=0\right]$ and the impurity interacts with the bath particles with a hard-core potential,

$$
V^{\text {int }}(r)= \begin{cases}\infty, & r \leqslant R \\ 0, & r>R\end{cases}
$$

It is important to recall that, in $2 \mathrm{D}$, the scattering amplitude depends logarithmically on momentum, so that the definition of the scattering length $a_{s}$ involves an arbitrary constant. Two alternative conventions are typically used. In the first one, $a_{s}$ is defined to fulfill $a_{s}=R$ for a hard-core potential, so that the two-body scattering wave function vanishes at $r=a_{s}$ [45] in analogy with the $3 \mathrm{D}$ case. This is the convention that we use in this work. With such a definition, the two-body binding energy for an attractive contact interaction is $\left|\epsilon_{b}\right|=4 \hbar^{2} /\left(m a_{s}^{2} e^{2 \gamma}\right)$, with $\gamma \simeq 0.577$ being Euler's constant $[46,47]$. Another definition of the $2 \mathrm{D}$ scattering length (now indicated by $b$ ) aims at maintaining a simple relation with the binding energy $\left|\epsilon_{b}\right|=$ $\hbar^{2} /\left(m b^{2}\right)$, in analogy with the 3D attractive problem $[43,48]$. The relation between the two conventions is $b=a_{s} e^{\gamma} / 2$.

For the hard-disk model, all the physics in the system is condensed into the gas parameter $n a_{s}^{2}$. We also notice that the closer $n a_{s}^{2}$ is to unity, the less this model is expected to faithfully describe the repulsive branch of the polaron, since coupling to molecular states is completely ignored.

\section{B. Dipolar model}

In the second model, all the particles in the system interact with each other through the same dipolar potential. We also consider all the dipoles to be polarized in the direction perpendicular to the plane of motion, so that the interaction between them is isotropic. Thus, the two potentials appearing in the Hamiltonian of Eq. (1) take the form

$$
V^{\text {bath }}(r)=V^{\text {int }}(r)=\frac{C_{d d}}{4 \pi} \frac{1}{r^{3}} .
$$

The purpose of including dipolar interparticle interaction between all the particles in the system, and not only between the bath and the impurity, is to study an experiment that could be suitable for current state-of-the-art experiments: a polarized system of fermionic polar atoms (such as ${ }^{161} \mathrm{Dy}$ or $\left.{ }^{167} \operatorname{Er}[35]\right)$, tightly confined in the polarization direction, with the majority of spin-up atoms forming the bath, and a vanishingly small concentration of spin-down impurity atoms. It would also be a good model in the case in which the impurity is an isotope of the same element, such as ${ }^{162}$ Dy into 
a bath of ${ }^{161}$ Dy. Differently from the short-range case, in the dipolar case the interaction between uneven fermions cannot be neglected.

For a dipolar system, the Hamiltonian (1) can be written in dimensionless form by expressing all distances in units of the characteristic length $r_{0}=m C_{d d} /\left(4 \pi \hbar^{2}\right)$ and energies in units of $\epsilon_{0}=\hbar^{2} / m r_{0}^{2}$. Hence, properties of the homogeneous system are governed by the dimensionless density $n r_{0}^{2}$ encoding the strength of the interactions, as done in previous works $[27,49,50]$. Although in three dimensions the dipoledipole potential is long ranged, in two dimensions it is not. Therefore, in the low-density regime it can be reduced to a contact interaction and we can use the gas parameter $n a_{s}^{2}$ for a better comparison with other potentials, such as the one in the previous subsection. In dipolar units, the scattering length has the value $a_{s}=r_{0} e^{2 \gamma}[50,51]$, so that $n a_{s}^{2} \simeq 10 n r_{0}^{2}$.

\section{METHOD}

We employ the diffusion Monte Carlo (DMC) method $[52,53]$ for finding the ground state of the Hamiltonian in Eq. (1). The DMC algorithm is a stochastic method that allows us to find the ground state of the system by propagation in imaginary time. For bosonic systems it gives exact results, within statistical errors, when the imaginary-time step tends to zero, $\delta \tau \rightarrow 0$, and for an infinite population of walkers $N_{W} \rightarrow \infty$ (a walker being a set of $N$ coordinates). In practice, convergence can be achieved using small imaginary-time steps and large enough number of walkers. It is well known that a trial wave function $\Psi_{T}$ used for importance sampling reduces the variance without introducing any additional bias, as long as $\Psi_{T}$ has a finite overlap with the exact groundstate wave function of the system. For fermionic systems, the wave function is not positive definite, giving rise to the so called sign problem. The most common technique to keep this problem under control is the fixed-node approximation. In this scheme, the DMC method is exact only if the nodal surface of $\Psi_{T}$ coincides with the one of the ground state. Otherwise, it becomes a variational method, which provides an upper bound for the ground-state energy.

In our simulations, we use a Jastrow-Slater trial wave function,

$$
\Psi_{T}(\mathbf{R})=\Psi_{A}\left(\mathbf{R}_{\uparrow}\right) \Psi_{J}(\mathbf{R}),
$$

where $\mathbf{R}=\left\{\mathbf{r}_{1}, \ldots, \mathbf{r}_{N_{\uparrow}}, \mathbf{r}_{\downarrow}\right\}$ is the set of all $N_{\uparrow}+1$ particle coordinates, and $\mathbf{R}_{\uparrow}$ is restricted to the $N_{\uparrow}$ particles of the bath. The antisymmetric wave function for the bath, $\Psi_{A}\left(\mathbf{R}_{\uparrow}\right)$, is a Slater determinant, where we use plane waves as singleparticle orbitals. These orbitals, which correspond to the nodal surface of the free Fermi gas, are accurate enough for the low densities considered here, as shown in a recent work [27].

The symmetric Jastrow part is written as

$$
\Psi_{J}(\mathbf{R})=\prod_{j=1}^{N_{\uparrow}} f_{\uparrow \downarrow}\left(r_{j \downarrow}\right) \prod_{i<j}^{N_{\uparrow}} f_{\uparrow \uparrow}\left(r_{i j}\right) .
$$

The two-body correlation functions $f_{\uparrow \uparrow}(r)$ and $f_{\uparrow \downarrow}(r)$ are constructed from the zero-energy two-body solution satisfying the conditions $f_{\uparrow \uparrow}(L / 2)=f_{\uparrow \downarrow}(L / 2)=1, f_{\uparrow \uparrow}^{\prime}(L / 2)=$ $f_{\uparrow \downarrow}^{\prime}(L / 2)=0$. For the dipolar model, the two-body solution is matched at a certain distance $r_{M}$ with a symmetrized phononic tail, reproducing the long distance behavior in the medium [49]. In general, the bath-bath and bath-impurity correlations are significantly different, so that in the dipolar model we consider different values of the matching distance $r_{M}$ for the two cases. Therefore we have two variational parameters: $r_{M}^{\uparrow \uparrow}$ and $r_{M}^{\uparrow \downarrow}$. For the hard-disk model, Jastrow correlations are implemented only for the impurity-bath pairs, since the bath is noninteracting $\left[f_{\uparrow \uparrow}(r)=1\right]$. In the latter case, the only variational parameter is $r_{H D} \leqslant L / 2$, at which we impose the conditions $f_{\uparrow \downarrow}\left(r_{H D}\right)=1, f_{\uparrow \downarrow}^{\prime}\left(r_{H D}\right)=0$.

In a DMC calculation, expectation values of a given operator $\hat{O}$ are obtained by sampling over the mixed probability distribution $f(\mathbf{R}, \tau)=\Psi_{T}(\mathbf{R}) \phi(\mathbf{R}, \tau)$. For a system of bosons, $\phi(\mathbf{R}, \tau)$ is the exact wave function of the system, while, for a fermionic system, it corresponds to the fixed-node upper bound related to the choice of the nodal surface. For long enough imaginary time, components of $\phi$ that are orthogonal to the ground state $\phi_{0}$ are removed and the only relevant contribution comes from $\phi_{0}$ :

$$
\langle\hat{O}\rangle_{\mathrm{DMC}}=\frac{\left\langle\Psi_{T}|\hat{O}| \phi_{0}\right\rangle}{\left\langle\Psi_{T} \mid \phi_{0}\right\rangle}=\lim _{\tau \rightarrow \infty} \frac{\int d \mathbf{R} \phi(\mathbf{R}, \tau) \hat{O} \Psi_{T}(\mathbf{R})}{\int d \mathbf{R} \phi(\mathbf{R}, \tau) \Psi_{T}(\mathbf{R})} .
$$

Equation (6) gives unbiased results when the operator $\hat{O}$ is the Hamiltonian or it commutes with $\hat{H}$. For diagonal operators that do not commute with the Hamiltonian, it is still possible to obtain exact values using the pure estimators technique [54]. In the case of nondiagonal operators, obtaining a pure estimator is more subtle. In this work, we will restrict our results to a first-order correction in $\Psi_{T}$ given by the extrapolated estimator

$$
\langle\hat{O}\rangle \simeq 2\langle\hat{O}\rangle_{\mathrm{DMC}}-\langle\hat{O}\rangle_{\mathrm{VMC}}
$$

where $\langle\hat{O}\rangle_{\mathrm{VMC}}=\frac{\left\langle\Psi_{T}|\hat{O}| \Psi_{T}\right\rangle}{\left\langle\Psi_{T} \mid \Psi_{T}\right\rangle}$ is the variational Monte Carlo (VMC) estimator. The above extrapolation is accurate when the DMC correction to the VMC result is small.

\section{RESULTS}

The QMC results that appear in this section will be compared with two approximate theories to benchmark them. This will be also of some utility to study the regime in which the system becomes universal in terms of the gas parameter. As a first approximation, we will compare our energies with the prediction that mean-field theory offers for the system [55]. On the other hand, we will also compare our results with a $T$-matrix study of the repulsive Fermi polaron [43]. The authors of Ref. [43] considered the ultradilute limit of spin-up impurities immersed in an spin-down bath, which is treated as an ideal Fermi gas. Quasiparticle properties (effective mass and quasi-particle residue) were then evaluated for both the attractive and the repulsive branches of a system where the impurity interacts with the bath via a short-range potential having scattering length $a_{s}$. Due to the similarity of the repulsive branch studied in that model with our hard-disk system described in Sec. II A, it is worthwhile to compare it with the results obtained with our QMC approach. 


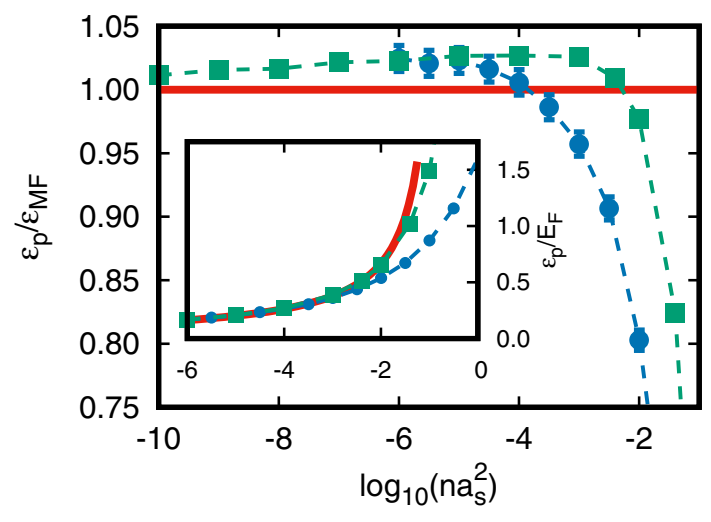

FIG. 1. Energy of the polaron in units of the mean-field energy in Eq. (9). The red line is the mean-field prediction, while green and blue symbols are DMC results for hard-disk and dipolar models, respectively. Dashed lines are guides to the eye. For large values of the gas parameter, the mean-field energy is not a good energy scale due to the logarithmic divergence of Eq. (9). Inset: polaron energy, in units of the bath Fermi energy $E_{\mathrm{F}}$, plotted for larger values of $n a_{s}^{2}$.

\section{A. Energy of the polaron}

The energy of the polaron is an important and experimentally accessible observable. It is defined as the energy difference between the pure system of $N_{\uparrow}$ particles and the same system with an added impurity, at fixed volume. Making use of this definition, it can be directly evaluated in QMC simulations as the chemical potential of the impurity; that is, it can be extracted from the difference between the energy of the system with an added impurity, $E\left(N_{\uparrow}, 1\right)$, and that of the pure system, $E\left(N_{\uparrow}, 0\right)$, at fixed volume:

$$
\varepsilon_{p}=\left[E\left(N_{\uparrow}, 1\right)-E\left(N_{\uparrow}, 0\right)\right]_{V} .
$$

In mean-field theory an expression for the 2D polaron energy can be obtained, valid in the limit of vanishing density:

$$
\varepsilon_{\mathrm{MF}}=\frac{4 \pi \hbar^{2} n}{m \ln \left(c_{0} n a_{s}^{2}\right)} .
$$

The dependence of the mean-field prediction (9) on a free parameter $c_{0}$ is a peculiarity of $2 \mathrm{D}$ systems that is related to the features of scattering theory in 2D [56]. This free parameter is related to a characteristic energy scale of the system [27,47]. In the present work, we set it to the value $c_{0}=e^{2 \gamma} \pi / 2 \simeq 4.98$, corresponding to using an energy scale equal to the Fermi energy $E_{\mathrm{F}}=2 \hbar^{2} \pi n / m$.

In Fig. 1, we show our QMC results compared to the mean-field prediction of Eq. (9). We plot the polaron energy in units of the mean-field energy, so that deviations from the mean field are enhanced. Although it is a good approximation, the mean field fails to accurately reproduce even the lower densities considered in this work, which is a well known fact in two-dimensional gases [57]. As the density is increased, the mean-field prediction has a logarithmic divergence and thus it does not stand as a good energy scale for values of $n a_{s}^{2}>$ $10^{-3}$. For this reason, in the inset of Fig. 1 we plot the polaron energy, for the highest gas parameters, in units of the Fermi energy, $E_{F}$. The error bars that appear in Fig. 1 include both statistical and systematic errors, the latter being the largest contribution. In the low density regime, the systematic error

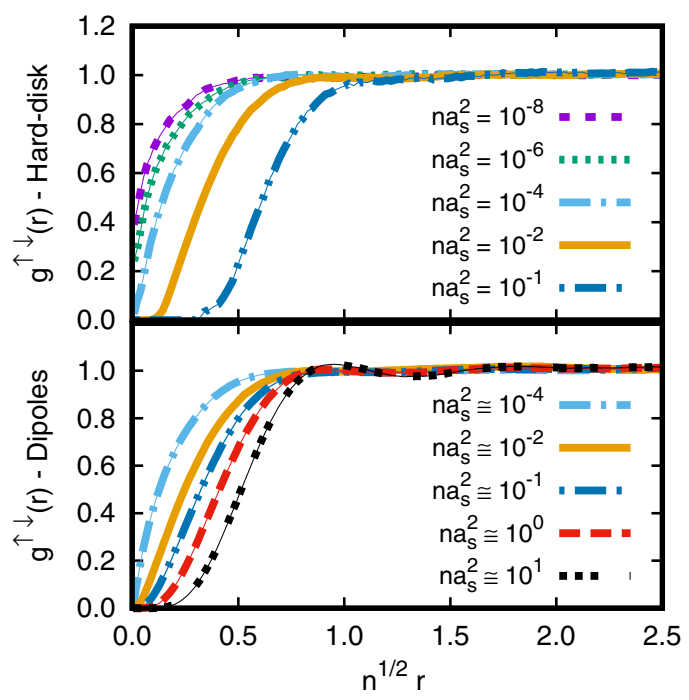

FIG. 2. Monte Carlo results for the pair distribution function $g^{\uparrow \downarrow}(r)$ between the impurity and the bath, evaluated for different values of the gas parameter $n a_{s}^{2}$ for the hard-disk model (top panel) and for the dipolar one (bottom panel).

is dominated by the finite value of the imaginary-time step $\delta \tau$, while for the higher densities the main source of error comes from finite-size effects. Concerning this latter issue, calculations have been done using 61 bath particles for all the dipolar system, while, for the hard-disk model, the exclusion of volume caused by the impurity makes it necessary to include 121 particles in the bath to keep finite-size effects under control when the gas parameter is higher than $n a_{s}^{2} \geqslant$ $10^{-2}$. In the case of hard-disk interaction, systematic errors for the polaron energy are of the order of $0.5 \%$, while for dipolar systems they grow up to $1 \%$.

\section{B. Pair distribution function}

The presence of the impurity affects the local properties of the bath. This effect can be analyzed by looking at the pair distribution function between the background and the impurity $g^{\uparrow \downarrow}(r)$, sometimes referred to as the density profile of the bath around the impurity. In DMC simulations, we can evaluate both this distribution function and the one involving bath particles, $g^{\uparrow \uparrow}(r)$ :

$$
\begin{aligned}
g^{\uparrow \uparrow}(r) & =\frac{2}{n N_{\uparrow}} \frac{\int d \mathbf{R} \phi_{0}(\mathbf{R}) \Psi_{T}(\mathbf{R}) \sum_{i<j}^{N_{\uparrow}} \delta\left(r-r_{i j}\right)}{\int d \mathbf{R} \phi_{0}(\mathbf{R}) \Psi_{T}(\mathbf{R})}, \\
g^{\uparrow \downarrow}(r) & =\frac{1}{n} \frac{\int d \mathbf{R} \phi_{0}(\mathbf{R}) \Psi_{T}(\mathbf{R}) \sum_{j=1}^{N_{\uparrow}} \delta\left(r-r_{\downarrow j}\right)}{\int d \mathbf{R} \phi_{0}(\mathbf{R}) \Psi_{T}(\mathbf{R})} .
\end{aligned}
$$

Figure 2 shows $g^{\uparrow \downarrow}(r)$, as a function of the dimensionless quantity $r \sqrt{n}$, for different gas parameters and for the two models considered in this work. The plot indicates that the hole around the impurity, arising from repulsive correlations between the impurity and bath particles, grows when the gas parameter is increased. We also notice that, at the lowest interaction strength shown for the dipolar model $\left(n a_{s}^{2} \simeq 10^{-4}\right)$, the distribution function closely resembles the one of the hard-disk model (except at distances compared to the core radius $R=a_{s}$ ) indicating the approaching to the low-density 


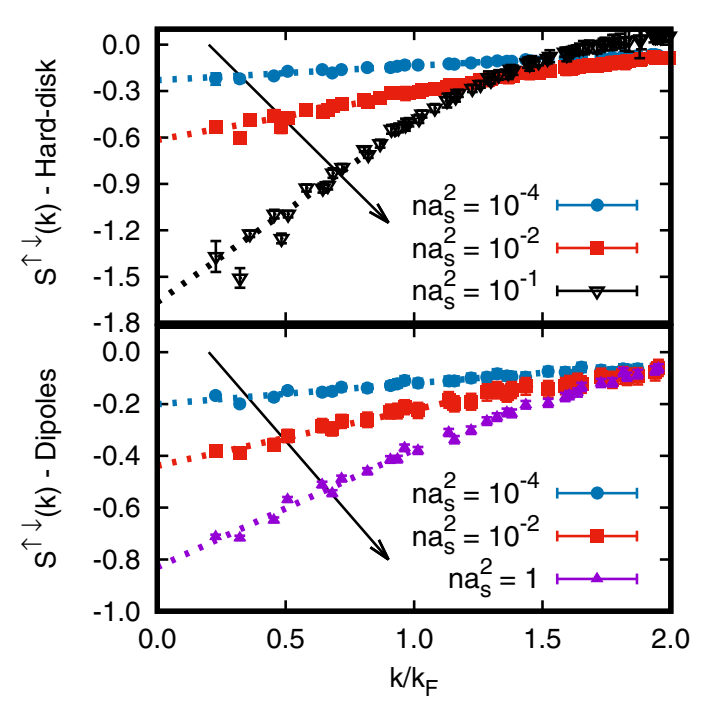

FIG. 3. Static structure factor $S^{\uparrow \downarrow}(k)$ involving correlations between the impurity and the bath particles, for small values of $k / k_{F}$, with $k_{F}=\sqrt{4 \pi n}$ being the Fermi momentum. Top (bottom) panel: results correspond to the hard-disk (dipolar) system for different values of the gas parameter. Same color and symbols are used to emphasize when the two models are evaluated at the same gas parameter. Dashed lines correspond to a linear extrapolation to $k \rightarrow$ 0 . The arrows indicate increasing density.

universal regime, similar to what one finds when comparing the polaron energies for the two models. For the dipolar model, the radial distributions have been evaluated using the pure estimators technique [54], while, for the hard-disk model, DMC results have been extrapolated as explained in the previous section [see Eq. (7)]. This also applies for the data in Fig. 3, and in both cases error bars are chosen to cover systematic errors.

As the gas parameter approaches $n a_{s}^{2} \simeq 1$, the radius of the hard-disk model starts to approach the mean interparticle distance and the model ceases to capture the physics of the repulsive branch with short-range interactions. Instead, the dipolar model still maintains its physical meaning in the highdensity regime and $g^{\uparrow \downarrow}(r)$ features Friedel oscillations, indicating the formation of shells of particles around the impurity. It is worth mentioning that all the radial distributions shown in Fig. 2 are evaluated in a system containing 61 bath particles except for the two highest densities shown for the hard-disk model $\left(n a_{s}^{2}=10^{-2}\right.$ and $\left.10^{-1}\right)$. In these latter cases, the large amount of volume excluded by the impurity enhances the finite-size effects and the use of 121 bath particles is needed to keep them under control.

Due to the interaction between the impurity and the medium as well as the statistics of the particles in the bath, the volume occupied by the impurity is different from the one of any of the bath particles. If one considers a mixture with a very low concentration of impurities, the total density of the mixture can be written at fixed pressure $P$ as $\rho(P, x)=\rho(P, x=0)(1+\alpha x)^{-1}$ with $x$ the concentration of the impurity atoms and $\alpha$ the excess volume parameter. As shown in [58,59], in the limit $x \rightarrow 0, \alpha$ can be approximately evaluated from the $k=0$ value of the static structure factor
$S^{\uparrow \downarrow}(k)$ correlating the impurity and the bath particles:

$$
S^{\uparrow \downarrow}(0)=-(1+\alpha),
$$

where $S^{\uparrow \downarrow}(k)$ is related to the Fourier transform of the radial distribution function $g^{\uparrow \downarrow}(r)$ :

$$
S^{\uparrow \downarrow}(k)=n \int d \mathbf{r} e^{i \mathbf{k} \cdot \mathbf{r}}\left[g^{\uparrow \downarrow}(r)-1\right] .
$$

The sign of $\alpha$ carries information on whether there is an excess or deficit of volume induced by the inclusion of the impurity particle in the bath: $\alpha>0(\alpha<0)$ indicates that the impurity occupies more (less) volume than a given bath particle. This quantity has been evaluated in condensed-matter systems, for example for an ${ }^{3} \mathrm{He}$ atom in bulk ${ }^{4} \mathrm{He}$. There, it was shown that the ${ }^{3} \mathrm{He}$ atom occupies near $30 \%$ more volume than the average volume occupied by the particles of the ${ }^{4} \mathrm{He}$ bath [8]. In that case, the increase of volume can be qualitatively explained in terms of the different zero-point motion that the two isotopes have, stemming from the mass difference.

For a system where all atoms have the same mass and the same interparticle interaction but where the species are distinguished by their spin component, as is the case of our dipolar system, a decrease of volume would arise because of Fermi statistics. In order to quantify this reduction, we evaluate the impurity-bath static structure factor of Eq. (13) for our system of dipoles at different densities (see bottom panel of Fig. 3). For this model, the volume coefficient $\alpha$ is negative for all the range of densities that we analyze, telling us that the impurity occupies less volume than one of the bath particles, since these are pushed further apart from each other due to Fermi repulsion. We see that the excess volume $\alpha$ decreases in magnitude with increasing density, that is, when the potential contributions to the energy start to be important compared to the Fermi repulsion. If one keeps increasing the density of the system up to the crystallization point $\left(n r_{0}^{2} \sim 50\right.$ [60]), the volume coefficient would approach zero $(\alpha \rightarrow 0)$, as it would for an impurity which is barely distinguishable from the bath atoms.

The deficit of volume can also be analyzed in our harddisk model (see top panel of Fig. 3). In this case, however, the physics is different from the dipolar model, where the only difference between the two species comes from Fermi statistics. In this model one has also to consider that the only interaction present in the system is that of the impurity with the ideal Fermi bath. As a result, two effects compete and dominate over each other in different regimes. For low values of the gas parameter, where the hard-core radius is small compared to the mean interparticle distance, one expects that all the deficit of volume would be caused by Fermi statistics, similar to the dipolar case. This is what can be seen when comparing the QMC results for the two models in Fig. 3: up to values of $n a_{s}^{2} \leqslant 10^{-4}$, the two interaction potentials give the same $\alpha$ parameter. In contrast, as the gas parameter increases and the system abandons the universal regime, the radius of the hard core starts to be compatible with the interparticle distance, and $\alpha$ is greater than that from the equivalent dipolar system. It is worth noticing that, for the highest gas parameter considered for this model, $n a_{s}^{2}=10^{-1}$, the volume coefficient becomes positive, meaning that the impurity, in this regime, 
occupies a bigger volume than an average particle in the ideal Fermi bath considered.

\section{Quasiparticle properties}

In the weakly interacting regime, one can assume that the wave function $\phi$ describing the state of the bath plus the impurity system has an important overlap with the state $\Phi^{\mathrm{NI}}$ in which interactions between the impurity and the bath are absent. The latter is a state representing a system containing a noninteracting impurity with momentum $k=0$ immersed in an unperturbed single-component bath. The quasiparticle residue $Z$ is defined through this overlap [61]:

$$
Z=\left|\left\langle\Phi^{\mathrm{NI}} \mid \phi\right\rangle\right|^{2} .
$$

For the system with hard-disk interaction, where the bath is an ideal Fermi gas, $\Phi^{\mathrm{NI}}$ reduces to $|\mathrm{FS}+1\rangle$, which stands for a Fermi sea with an added noninteracting impurity at zero momentum. In our dipolar model, in contrast, bath particles interact with each other, so that $\Phi^{\mathrm{NI}}$ is the state of the interacting bath with the addition of a noninteracting impurity at zero momentum. The quasiparticle residue in Eq. (14) also represents the probability of free propagation of the impurity in the medium.

In the theory of Fermi liquids, the quasiparticle residue $Z$ corresponds to the jump in the momentum distribution $n(k)$ at the Fermi momentum. In our study, if we consider the impurity as the zero-density limit of a Fermi sea, we obtain the relation $Z=n_{\downarrow}(k=0)-n_{\downarrow}\left(k=0^{+}\right)$. The components at $k>0$ scale with the inverse volume, so that they are negligible in the thermodynamic limit for the bath $[62,63]$. In QMC simulations in real space, the quasiparticle residue is best extracted from the Fourier transform of the momentum distribution, the one-body density matrix (OBDM). While its integral over volume would yield $n_{\downarrow}(k=0)$ for a finite system, its asymptotic value at $r \rightarrow L / 2$ is a better estimate of $Z$, since the finite-size component is automatically removed. Following this scheme, we evaluate the quasiparticle residue from the asymptotic behavior of the OBDM involving the impurity, which in the DMC framework is evaluated from the following estimator:

$$
Z=\lim _{\left|\mathbf{r}_{\downarrow}^{\prime}-\mathbf{r}_{\downarrow}\right| \rightarrow L / 2}\left\langle\frac{\Psi_{T}\left(\mathbf{R}_{\uparrow}, \mathbf{r}_{\downarrow}^{\prime}\right)}{\Psi_{T}\left(\mathbf{R}_{\uparrow}, \mathbf{r}_{\downarrow}\right)}\right\rangle .
$$

Since this DMC estimator is nondiagonal, the result is generally biased due to the choice of the trial wave function (cf. Sec. III). Our estimation is based on the extrapolated estimator in Eq. (7), which we expect to be accurate enough due to the quality of the trial wave function, especially at low densities. In Fig. 4, we show our results for the residue $Z$, following the prescription of Eq. (15), for both hard disks and dipoles. We find that a universal regime can be identified for gas parameters lower than $n a_{s}^{2}<10^{-3}$, up to where relative differences between the quasiparticle residues evaluated for the two models remain below 5\%. These relative deviations are comparable to the ones reported for the polaron energy at that same gas parameter in Sec. IV A. However, in the regime $n a_{s}^{2}>10^{-3}$, clear differences between the two models appear: for the dipolar model the quasiparticle residue features values higher than 0.6 in all the interval of $n a_{s}^{2}$ considered here. In
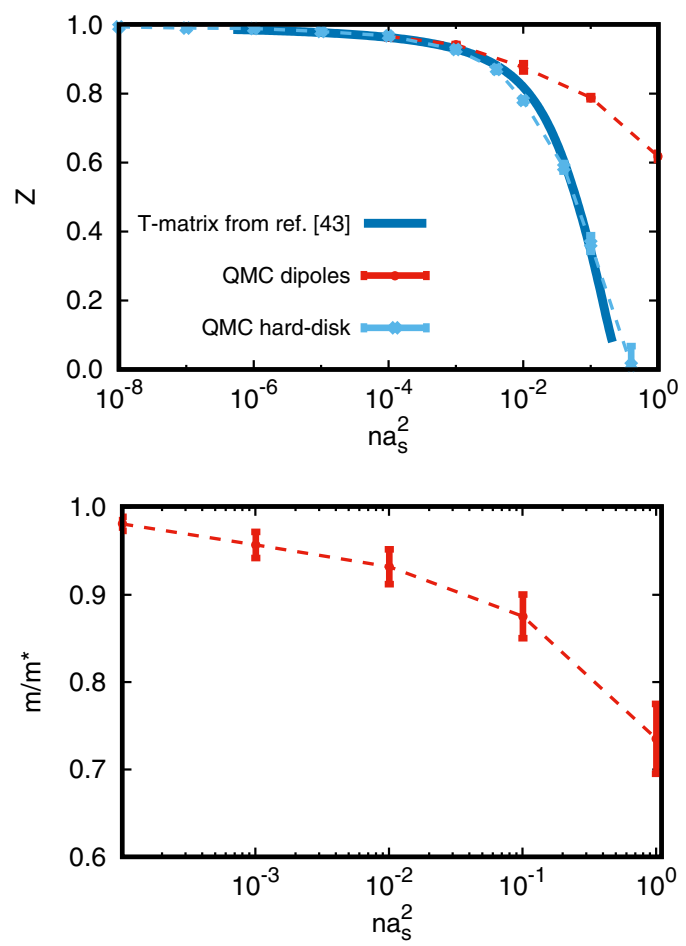

FIG. 4. Quasiparticle residue $Z$ (top panel) and effective mass of the polaron (bottom panel) as a function of the gas parameter $n a_{s}^{2}$. Red symbols correspond to he dipolar system, blue ones correspond to the hard-core impurity, and the solid blue line shows the manybody $T$-matrix theory results [43]. Dashed lines are guides to the eye.

contrast, for the hard-disk model, $Z$ is highly suppressed as the gas parameter is increased, reflecting that the interaction radius begins to be comparable to the interparticle distance, making it difficult for the impurity to perform a free displacement. Noticeably, for the largest value of the gas parameter $\left(n a_{s}^{2}=4 \times 10^{-1}\right)$ the residue almost vanishes, suggesting a tendency of the impurity to get localized as the interaction strength becomes very large. In the same plot, we include the $T$-matrix results from Ref. [43], corresponding to the quasiparticle residue of the repulsive branch of the 2D Fermi impurity problem with short-range interactions. These results are in reasonable agreement with our hard-disk impurity model, up to a regime where the excited repulsive polaron loses its identity due to strong coupling to the molecular branch.

The other quantity that is of relevance for studying the polaron in a quasiparticle picture is its effective mass, that is, the mass of the quasi-particle formed by the impurity "dressed" by the medium. In a DMC simulation, the effective mass $m^{*}$ is obtained from the asymptotic diffusion coefficient in imaginary time of the impurity throughout the bath $[8,16]$,

$$
\frac{m}{m^{*}}=\lim _{\tau \rightarrow \infty} \frac{1}{4 \tau} \frac{D_{s}^{\downarrow}(\tau)}{D_{0}},
$$

with $D_{0}=\frac{\hbar^{2}}{2 m}$ being the free-particle diffusion constant and $D_{s}^{\downarrow}(\tau)=\left\langle\left[\mathbf{r}_{\downarrow}(\tau)-\mathbf{r}_{\downarrow}(0)\right]^{2}\right\rangle$ the squared imaginary-time displacement of the impurity. In the bottom panel of Fig. 4 
we report our DMC results for the dipolar system, which show that interaction effects increase the effective mass of the polaron by roughly $30 \%$ as the gas parameter increases up to $n a_{s}^{2} \sim 1$. Although the effective mass for the hard-disk model has not been evaluated in the present work, we expect it to be in agreement with the one in Ref. [43], similarly to what happens with the quasiparticle residue. When compared to the data for short-range interactions from Ref. [43] (not shown), the effective mass of the dipolar model appears to be less affected by interactions and remains closer to its noninteracting limit $\left(m^{*}=m\right)$, in analogy with what is observed for the quasi-particle residue.

It is worth noticing that, through the knowledge of the effective mass, we can also access the excitation spectrum of the polaron at low momenta,

$$
\epsilon_{p}(k)=\epsilon_{p}(k=0)+\frac{\hbar^{2}}{2 m^{*}} k^{2}+O\left(k^{4}\right),
$$

where $\epsilon_{p}(k=0)$ is the chemical potential of the polaron discussed in Sec. IV A.

\section{DISCUSSION AND CONCLUSIONS}

By means of the DMC method, we have calculated the energetic and structural properties of a repulsive polaron in a 2D polarized Fermi system. The use of two different interparticle interactions between the polaron and the bath allows us to analyze the range of universality of the polaron properties in terms of the gas parameter $n a_{s}^{2}$. We show that the polaron energy is universal up to $n a_{s}^{2} \approx 10^{-5}$; beyond this regime, it depends on the specific shape of the interaction. Note that our two models also differ for the bath properties (noninteracting vs interacting), which could contribute to this nonuniversality.

The presence of a polaron also affects local properties of the bath. The estimation of the two-body radial distribution functions and of the static structure factor helps quantify this effect. The limit $k \rightarrow 0$ of the static structure factor gives the excess volume coefficient. Our results for the dipolar model show that the effective volume occupied by the impurity shrinks with respect to the one of a particle in the bath. The reason underlying this result is the lack of Fermi correlations between the polaron and the medium. On the other hand, for the hard-disk model, in the regime where the exclusion of volume caused by the potential dominates over the Fermi repulsion between bath particles, the volume coefficient becomes positive.

In the weakly interacting regime, where the effective mass is close to the bare mass of the impurity and the quasiparticle residue is the main contribution to the ground state wave function, the quasiparticle picture is valid. This allows us to approximately describe the problem as a quasiparticle made up of the impurity "dressed" by the interactions with the bath, propagating through the medium with a definite effective mass that takes into account interaction effects. When the $Z$ residue starts to depart significantly from 1 , the quasiparticle picture is not able to describe completely the many-body physics involved in the problem.

Recent experimental data [64] have been reported for the same system explored here. However, the values of the gas parameter at which those measurements have been carried out are larger than the universality limit determined in this work. Therefore, finite-range effects should be taken into account in future theoretical studies to allow for a quantitative comparison with experiments. One can expect that, by fixing both the $s$-wave scattering length and the effective range, it would be possible to extend the regime of universality to larger values of the gas parameter. This extension of the regime of universality was recently reported for ultradilute Bose-Bose mixtures [65]

Data and additional details about the numerical simulations are made publicly available [66].

\section{ACKNOWLEDGMENTS}

We acknowledge Richard Schmidt for providing the data from Ref. [43]. This work has been supported by the Ministerio de Economia, Industria y Competitividad (MINECO, Spain) under Grant No. FIS2017-84114-C2-1-P, FPI fellowship BES2015-074088, and by Provincia Autonoma di Trento. G.B. acknowledges D.E. Galli for access to computational resources at the Department of Physics of the University of Milan.
[1] L. D. Landau, Über Die Bewegung der Elektronen in Kristallgitter gitter, Phys. Z. Sowjetunion 3, 644 (1933).

[2] L. D. Landau and S. I. Pekar, Effective mass of a polaron, Zh. Eksp. Teor. Fiz. 18, 419 (1948).

[3] H. Fröhlich, Electrons in lattice fields, Adv. Phys. 3, 325 (1954).

[4] R. P. Feynman, Slow electrons in a polar crystal, Phys. Rev. 97, 660 (1955).

[5] N. V. Prokof'ev and B. V. Svistunov, Polaron Problem by Diagrammatic Quantum Monte Carlo, Phys. Rev. Lett. 81, 2514 (1998).

[6] A. S. Mishchenko, N. V. Prokof'ev, A. Sakamoto, and B. V. Svistunov, Diagrammatic quantum Monte Carlo study of the Fröhlich polaron, Phys. Rev. B 62, 6317 (2000).

[7] D. O. Edwards and M. S. Pettersen, Lectures on the properties of liquid and Solid ${ }^{3} \mathrm{He}-{ }^{4} \mathrm{He}$ mixtures at low temperatures, J. Low Temp. Phys. 87, 473 (1992).
[8] J. Boronat and J. Casulleras, Quantum Monte Carlo study of static properties of one ${ }^{3} \mathrm{He}$ atom in superfluid ${ }^{4} \mathrm{He}$, Phys. Rev. B 59, 8844 (1999).

[9] R.F. Bishop, The binding energy of a $\Lambda$-particle in nuclear matter: A comparison of two formulations, Nucl. Phys. B 17, 573 (1970).

[10] J. Tempere, W. Casteels, M. K. Oberthaler, S. Knoop, E. Timmermans, and J. T. Devreese, Feynman path-integral treatment of the BEC-impurity polaron, Phys. Rev. B 80, 184504 (2009).

[11] F. M. Cucchietti and E. Timmermans, Strong-Coupling Polarons in Dilute Gas Bose-Einstein Condensates, Phys. Rev. Lett. 96, 210401 (2006).

[12] N. B. Jørgensen, L. Wacker, K. T. Skalmstang, M. M. Parish, Jesper Levinsen, Rasmus S. Christensen, G. M. Bruun, and J. J. Arlt, Observation of Attractive and Repulsive Polarons 
in a Bose-Einstein Condensate, Phys. Rev. Lett. 117, 055302 (2016).

[13] M. G. Hu, M. J. Van de Graaff, D. Kedar, J. P. Corson, E. A. Cornell, and D. S. Jin, Bose Polarons in the Strongly Interacting Regime, Phys. Rev. Lett. 117, 055301 (2016).

[14] Z. Z. Yan, Y. Ni, C. Robens, and M. W. Zwierlein, Bose polarons near quantum criticality, arXiv:1904.02685.

[15] S. P. Rath and R. Schmidt, Field-theoretical study of the Bose polaron, Phys. Rev. A 88, 053632 (2013).

[16] L. A. P. Ardila and S. Giorgini, Impurity in a Bose-Einstein condensate: Study of the attractive and repulsive branch using quantum Monte Carlo methods, Phys. Rev. A 92, 033612 (2015).

[17] A. Schirotzek, C. H. Wu, A. Sommer, and M. W. Zwierlein, Observation of Fermi Polarons in a Tunable Fermi Liquid of Ultracold Atoms, Phys. Rev. Lett. 102, 230402 (2009).

[18] C. Kohstall, M. Zaccanti, M. Jag, A. Trenkwalder, P. Massignan, G. M. Bruun, F. Schreck, and R. Grimm, Metastability and coherence of repulsive polarons in a strongly interacting Fermi mixture, Nature (London) 485, 615 (2012).

[19] N. Prokof'ev and B. Svistunov, Fermi-polaron problem: Diagrammatic Monte Carlo method for divergent sign-alternating series, Phys. Rev. B 77, 020408(R) (2008).

[20] S. Nascimbène, N. Navon, K. J. Jiang, L. Tarruell, M. Teichmann, J. McKeever, F. Chevy, and C. Salomon, Collective Oscillations of an Imbalanced Fermi Gas: Axial Compression Modes and Polaron Effective Mass, Phys. Rev. Lett. 103, 170402 (2009).

[21] P. Massignan, M. Zaccanti, and G. M. Bruun, Polarons, dressed molecules and itinerant ferromagnetism in ultracold Fermi gases, Rep. Prog. Phys. 77, 034401 (2014).

[22] M. W. Zwierlein, A. Schirotzek, C. H. Schunck, and W. Ketterle, Fermionic superfluidity with imbalanced spin populations, Science 311, 492 (2006).

[23] G. B. Partridge, W. Li, R. I. Kamar, Y. A. Liao, and R. G. Hulet, Pairing and phase separation in a polarized Fermi gas, Science 311, 503 (2006).

[24] S. Pilati, G. Bertaina, S. Giorgini, and M. Troyer, Itinerant Ferromagnetism of a Repulsive Atomic Fermi Gas: A Quantum Monte Carlo Study, Phys. Rev. Lett. 105, 030405 (2010).

[25] G.B. Jo, Y. R. Lee, J. H. Choi, C. A. Christensen, T. H. Kim, J. H. Thywissen, D. E. Pritchard, and W. Ketterle, Itinerant ferromagnetism in a Fermi gas of ultracold atoms, Science 325, 1521 (2009).

[26] G. Valtolina, F. Scazza, A. Amico, A. Burchianti, A. Recati, T. Enss, M. Inguscio, M. Zaccanti, and G. Roati, Exploring the ferromagnetic behaviour of a repulsive Fermi gas through spin dynamics, Nat. Phys. 13, 704 (2017).

[27] T. Comparin, R. Bombín, M. Holzmann, F. Mazzanti, J. Boronat, and S. Giorgini, Two-dimensional mixture of dipolar fermions: Equation of state and magnetic phases, Phys. Rev. A 99, 043609 (2019).

[28] M. Nakagawa and N. Kawakami, Laser-Induced Kondo Effect in Ultracold Alkaline-Earth Fermions, Phys. Rev. Lett. 115, 165303 (2015).

[29] J. Stuhler, A. Griesmaier, T. Koch, M. Fattori, T. Pfau, S. Giovanazzi, P. Pedri, and L. Santos, Observation of DipoleDipole Interaction in a Degenerate Quantum Gas, Phys. Rev. Lett. 95, 150406 (2005).
[30] A. Griesmaier, J. Werner, S. Hensler, J. Stuhler, and T. Pfau, Bose-Einstein Condensation of Chromium, Phys. Rev. Lett. 94, 160401 (2005).

[31] M. Lu, N. Q. Burdick, S. H. Youn, and B. L. Lev, Strongly Dipolar Bose-Einstein Condensate of Dysprosium, Phys. Rev. Lett. 107, 190401 (2011).

[32] M. Lu, N. Q. Burdick, and B. L. Lev, Quantum Degenerate Dipolar Fermi Gas, Phys. Rev. Lett. 108, 215301 (2012).

[33] K. Aikawa, A. Frisch, M. Mark, S. Baier, A. Rietzler, R. Grimm, and F. Ferlaino, Bose-Einstein Condensation of Erbium, Phys. Rev. Lett. 108, 210401 (2012).

[34] K. Aikawa, A. Frisch, M. Mark, S. Baier, R. Grimm, and F. Ferlaino, Reaching Fermi Degeneracy Via Universal Dipolar Scattering, Phys. Rev. Lett. 112, 010404 (2014).

[35] A. Trautmann, P. Ilzhöfer, G. Durastante, C. Politi, M. Sohmen, M. J. Mark, and F. Ferlaino, Dipolar Quantum Mixtures of Erbium and Dysprosium Atoms, Phys. Rev. Lett. 121, 213601 (2018).

[36] M. Wenzel, T. Pfau, and I. Ferrier-Barbut, A fermionic impurity in a dipolar quantum droplet, Phys. Scr. 93, 104004 (2018).

[37] B. Kain and H. Y. Ling, Polarons in a dipolar condensate, Phys. Rev. A 89, 023612 (2014).

[38] L. A. Peña Ardila and T. Pohl, Ground-state properties of dipolar bose polarons, J. Phys. B 52, 015004 (2018).

[39] N. Matveeva and S. Giorgini, Impurity Problem in a Bilayer System of Dipoles, Phys. Rev. Lett. 111, 220405 (2013).

[40] J. Vlietinck, J. Ryckebusch, and K. Van Houcke, Diagrammatic Monte Carlo study of the Fermi polaron in two dimensions, Phys. Rev. B 89, 085119 (2014).

[41] M. Koschorreck, D. Pertot, E. Vogt, B. Fröhlich, M. Feld, and M. Köhl, Attractive and repulsive Fermi polarons in two dimensions, Nature (London) 485, 619 (2012).

[42] B. Fröhlich, M. Feld, E. Vogt, M. Koschorreck, W. Zwerger, and M. Köhl, Radio-Frequency Spectroscopy of a Strongly Interacting Two-Dimensional Fermi Gas, Phys. Rev. Lett. 106, 105301 (2011).

[43] R. Schmidt, T. Enss, V. Pietilä, and E. Demler, Fermi polarons in two dimensions, Phys. Rev. A 85, 021602(R) (2012).

[44] V. Ngampruetikorn, J. Levinsen, and Meera M. Parish, Repulsive polarons in two-dimensional Fermi gases, Europhys. Lett. 98, 30005 (2012).

[45] S. Pilati, J. Boronat, J. Casulleras, and S. Giorgini, Quantum Monte Carlo simulation of a two-dimensional Bose gas, Phys. Rev. A 71, 023605 (2005).

[46] G. Bertaina and S. Giorgini, BCS-BEC Crossover in a TwoDimensional Fermi Gas, Phys. Rev. Lett. 106, 110403 (2011).

[47] G. Bertaina, Two-dimensional short-range interacting attractive and repulsive Fermi gases at zero temperature, Eur. Phys. J. Spec. Top 217, 153 (2013).

[48] D. S. Petrov and G. V. Shlyapnikov, Interatomic collisions in a tightly confined Bose gas, Phys. Rev. A 64, 012706 (2001).

[49] G. E. Astrakharchik, J. Boronat, I. L. Kurbakov, and Yu E. Lozovik, Quantum Phase Transition in a Two-Dimensional System of Dipoles, Phys. Rev. Lett. 98, 060405 (2007).

[50] A. Macia, F. Mazzanti, J. Boronat, and R. E. Zillich, Microscopic description of anisotropic low-density dipolar Bose gases in two dimensions, Phys. Rev. A 84, 033625 (2011).

[51] C. Ticknor, Two-dimensional dipolar scattering, Phys. Rev. A 80, 052702 (2009). 
[52] B. L. Hammond, W. A. Lester, and P. J. Reynolds, Monte Carlo Methods in Ab Initio Quantum Chemistry (World Scientific, Singapore, 1994).

[53] I. Kosztin, B. Faber, and K. Schulten, Introduction to the diffusion Monte Carlo method, Am. J. Phys. 64, 633 (1996).

[54] J. Casulleras and J. Boronat, Unbiased estimators in quantum Monte Carlo methods: Application to liquid ${ }^{4} \mathrm{He}$, Phys. Rev. B 52, 3654 (1995).

[55] M. Schick, Two-dimensional system of hard-core bosons, Phys. Rev. A 3, 1067 (1971).

[56] L. Pitaevskii and S. Stringari, Bose-Einstein Condensation and Superfluidity (Oxford University Press, Oxford, 2016).

[57] G. E. Astrakharchik, J. Boronat, J. Casulleras, I. L. Kurbakov, and Yu. E. Lozovik, Equation of state of a weakly interacting two-dimensional Bose gas studied at zero temperature by means of quantum Monte Carlo methods, Phys. Rev. A 79, 051602(R) (2009).

[58] M. Saarela and E. Krotscheck, Hydrogen isotope and ${ }^{3} \mathrm{He}$ impurities in liquid ${ }^{4} \mathrm{He}$, J. Low Temp. Phys. 90, 415 (1993).

[59] K. E. Kürten and M. L. Ristig, Atomic impurities in liquid helium, Phys. Rev. B 27, 5479 (1983).
[60] N. Matveeva and S. Giorgini, Liquid and Crystal Phases of Dipolar Fermions in two Dimensions, Phys. Rev. Lett. 109, 200401 (2012).

[61] J. Vlietinck, J. Ryckebusch, and K. Van Houcke, Quasiparticle properties of an impurity in a Fermi gas, Phys. Rev. B 87, 115133 (2013).

[62] M. Punk, P. T. Dumitrescu, and W. Zwerger, Polaron-tomolecule transition in a strongly imbalanced Fermi gas, Phys. Rev. A 80, 053605 (2009).

[63] A. Guidini, G. Bertaina, D. E. Galli, and P. Pieri, Condensed phase of Bose-Fermi mixtures with a pairing interaction, Phys. Rev. A 91, 023603 (2015).

[64] N. Darkwah Oppong, L. Riegger, O. Bettermann, M. Höfer, J. Levinsen, M. M. Parish, I. Bloch, and S. Fölling, Observation of Coherent Multiorbital Polarons in a Two-Dimensional Fermi Gas, Phys. Rev. Lett. 122, 193604 (2019).

[65] V. Cikojević, L. Vranješ Markić, G. E. Astrakharchik, and J. Boronat, Universality in ultradilute liquid bose-bose mixtures, Phys. Rev. A 99, 023618 (2019).

[66] R. Bombín, T. Comparin, G. Bertaina, J. Boronat, F. Mazzanti, and S. Giorgini, Data two dimensional Fermi polaron, Zenodo (2019), doi: 10.5281/zenodo.3236022. 